\title{
Note on Intuitionistic N-Closed Sets
}

\author{
${ }^{1}$ M. Lellis Thivagar $\&{ }^{2}$ M. Anbuchelvi \\ ${ }^{I}$ School Of Mathematics Madurai Kamaraj University, Madurai-625021.Tamil Nadu, INDIA \\ .$^{2}$ Department of Mathematics, V.V.Vanniaperumal College For Women, Virudhunar-626001. Tamil Nadu, \\ INDIA.
}

Abstract: In this paper we introduce and investigate intuitionistic $N$-closed sets and Intuitionistic almost regular space in a intuitionistic topological spaces.

Key words and Phrases: int (A), el (A), intuitionistic almost regular spaces, intuitionistic $N$-closed sets., AMS subject classification 2010:57D05.

\section{Introduction}

First, D.Coker et al [2] introduced intuitionistic fuzzy topological spaces, intuitionistic topological spaces and the concept of Compactness on Intuitionistic topological spaces.In this paper we introduce and investigate intuitionistic Almost regular spaces and intuitionistic N-closed sets on intuitionistic topological spaces.Also we investigate their properties via intutionistic $T_{2}(i)$-spaces.

\section{Preliminaries}

Throughout this paper $(\tilde{X}, \tilde{\tau})$ (or briefly $\tilde{X}$ ) represent intuitionistic topological space on which no separation axioms are assumed unless explicitly stated.

Let us recall the following definitions, which are useful in the sequel.

Definition II.1. [1] Let $X$ be a nonempty set.An intuitionistic set $A$ is an object of the form $A=\left(A_{1}, A_{2}\right)$ where $A_{1}$ and $A_{2}$ are disjoint subsets of $X$. The set $A_{1}$ is the set of all members of $A$ and $A_{2}$ is the set of all non-members of $A$.

Definition II.2. [1] Let $X$ be a nonempty set, $a \in X$ and $A=\left(A_{1}, A_{2}\right)$ be an intuitionistic subset of $X$. Intuitionistic set $\tilde{a}=\left(\{a\},\{a\}^{c}\right)$ is called an intuitionistic point in $X$. The intuitionistic point $\tilde{a} \in A$ iff $a \in A_{1}$.

Definition II.3. [1] Let $X$ be a nonempty set. $A=\left(A_{1}, A_{2}\right), B=\left(B_{1}, B_{2}\right)$ and $\left\{A_{i}=\left(A_{i}^{(1)}, A_{i}^{(2)}\right) / i \in I\right\}$ are intuitionistic subsets of $X$. Then
(i) $A \subseteq B$ iff $A_{1} \subseteq B_{1}$ and $B_{2} \subseteq A_{2}$.
(ii) $A=B$ iff $A \subseteq B$ and $B \subseteq A$.
(iii) $A^{c}=\left(A_{2}, A_{1}\right)$.
(iv) $\bigcap A_{i}=\left(\bigcap A_{i}^{(1)}, \bigcup A_{i}^{(2)}\right)$.
(v) $\bigcup A_{i}=\left(\bigcup A_{i}^{(1)}, \bigcap A_{i}^{(2)}\right)$.
(vi) $\tilde{\varnothing}=(\varnothing, X)$.
(vii) $\tilde{X}=(X, \varnothing)$.

Definition II.4. [2] An intuitionistic topology on a nonempty set $X$ is a family $\tilde{\tau}$ of intuitionistic sets in $X$ containing $\Phi, \tilde{X}$ and closed under finite infima and arbitrary suprema.

Then the pair $(\tilde{X}, \tilde{\tau})$ is called an intuitionistic topological space.Every member of $\tilde{\tau}$ is known as an intuitionistic open set in $\tilde{X}$ The complement $A^{c}$ of an intuitionistic open set $A$ is called intuitionistic closed set in $\tilde{X}$.

Definition II.5. [2] Let $X$ be a nonempty set and let $A$ be an intuitionistic subset of X. Then the intuitionistic 
interior and intuitionistic closure of $A$ is defined by

(i). $\operatorname{int}(A)=\bigcup\{U: U$ is an intuitionistic open set of $\tilde{X}$ and $U \subseteq A\}$.

(ii). $\overline{e l}(\mathrm{~A})=\bigcap\{\mathrm{F}: \mathrm{F}$ is an intutionistic closed set of $\tilde{X}$ and $\mathrm{A} \subseteq \mathrm{F}\}$.

For any intutionistic subset $\mathrm{A}$ of $\mathrm{X}, \operatorname{int}\left(\mathrm{A}^{\mathrm{c}}\right)=[\mathrm{el}(\mathrm{A})]^{\mathrm{c}}$ and $\mathrm{el}\left(\mathrm{A}^{\mathrm{c}}\right)=[\operatorname{int}(\mathrm{A})]^{\mathrm{c}}$.

Definition II.6.[2] Let $\mathrm{f}:(\tilde{X}, \tilde{\tau}) \rightarrow(\tilde{Y}, \tilde{\sigma})$ be a function .If $\mathrm{A}=\left(\mathrm{A}_{1}, \mathrm{~A}_{2}\right)$ is an intutionistic subset of $\mathrm{X}$, then the image of $A$ under $f$, denoted by $f(A)$, is an intutionistic subset of $Y$ and defined by $f(A)=\left(f\left(A_{1}\right), f-\left(A_{2}\right)\right)$, where $\mathrm{f}-\left(\mathrm{A}_{2}\right)=\left(\mathrm{f}\left(\left(\mathrm{A}_{2}\right)^{\mathrm{c}}\right)\right)^{\mathrm{c}}$.

Definition II.7.[2] Let $\mathrm{f}:(\tilde{X}, \tilde{\tau}) \rightarrow(\tilde{Y}, \tilde{\sigma})$ be a function.If $\mathrm{B}=\left(\mathrm{B}_{1}, \mathrm{~B}_{2}\right)$ is an intutionistic subset of $\mathrm{Y}$, then the pre-image of $B$ under $f$, denoted by $f^{-1}(B)$ is an intutionistic subset of $X$ and defined by $f^{-1}(B)=\left(f^{1}\left(B_{1}\right), f^{-}\right.$ $\left.{ }^{1}\left(\mathrm{~B}_{2}\right)\right)$.

Definition II.8.[2] Let $(\tilde{X}, \tilde{\tau})$ be a intutionistic topological space.

(i) If a family $\left\{\mathrm{G}_{\mathrm{i}}=\left\langle\mathrm{G}_{\mathrm{i}}^{1}, \mathrm{G}_{\mathrm{i}}^{2}\right\rangle: \mathrm{i} \in \mathrm{J}\right\}$ of intutionistic open sets in $\mathrm{X}$ satisfies the condition $\bigcup_{i \in J} G_{i}=X^{\text {, }}$ then it is called an intutionistic open cover of $\tilde{X}$. A finite subfamily of intutionistic open cover $\left\{G_{i}=\left\langle G^{1}\right.\right.$ , $\left.\mathrm{G}^{2}>: \mathrm{i} \in \mathrm{J}\right\}$ of $\tilde{X}$, which is also an open cover of $\tilde{X}$, is called an intutionistic finite sub cover of $\tilde{X}$.

(ii) A family $\left\{\mathrm{F}_{\mathrm{i}}=\left\langle\mathrm{F}_{\mathrm{i}}^{1}, \mathrm{~F}^{2}{ }_{\mathrm{i}}\right\rangle: \mathrm{i} \in \mathrm{J}\right\}$ of intuitionistic closed sets in $\mathrm{X}$ satisfies the finite intersection property (briefly FIP) iff every finite subfamily $\left\{\mathrm{F}_{\mathrm{i}}: \mathrm{i}=1,2, \ldots \mathrm{n}\right\}$ of $\left\{\mathrm{F}_{\mathrm{i}}=\left\langle\mathrm{F}_{\mathrm{i}}^{1}, \mathrm{~F}_{\mathrm{i}}{ }_{\mathrm{i}}\right\rangle: \mathrm{i} \in \mathrm{J}\right\}$ satisfies the condition $\bigcap_{i=1}^{n} F_{i} \neq \Phi$.

Definition II.9.[2] An intutionistic topological space $(\tilde{X}, \tilde{\tau})$ is said to be intutionistic compact iff every intutionistic open cover $\left\{\mathrm{G}_{\mathrm{i}}=\left\langle\mathrm{G}_{\mathrm{i}}^{1}, \mathrm{G}_{\mathrm{i}}^{2}\right\rangle: \mathrm{i} \in \mathrm{J}\right\}$ of $\tilde{X}$ has a intutionistic finite subcover.

Definition II.10.[2]An intutionistic topological space $(\tilde{X}, \tilde{\tau})$ is said to be intutionistic nearly compact iff every $\left\{\mathrm{G}_{\mathrm{i}}=\left\langle\mathrm{G}_{\mathrm{i}}^{1}, \mathrm{G}_{\mathrm{i}}^{2}\right\rangle: \mathrm{i} \in \mathrm{J}\right\}$ of $\tilde{X}$, there exists a finite set $J_{0}$ of $J$ such that $\bigcup_{i \in J_{0}} \operatorname{int}\left(\operatorname{el}\left(G_{i}\right)\right)=邓$.

III. Intuitionistic $N$-Closed Sets.

Proposition III.1. [2] Let $(\tilde{X}, \tilde{\tau})$ be a intuitionistic topological space.

(i). If a family $\left\{G_{i}=<G_{i}^{1}, G_{i}^{2}>: i \in J\right\}$ of intuitionistic open sets in $(\tilde{X}, \tilde{\tau})$ satisfies the condition $A \subseteq \bigcup_{i \in J} G_{i}$ then it is an intuitionistic open cover of $A$.

(ii).Let $\left\{G_{i}=<G_{i}^{1}, G_{i}^{2}>: i \in J\right\}$ be a family of intuitionistic open sets in $(\tilde{X}, \tilde{\tau})$, which covers $A$. If there exists a finite subset $J_{0}$ of $J$ such that $A \subseteq \bigcup_{i \in J_{0}} G_{i}$ then $\left\{G_{i}=<G_{i}^{1}, G_{i}^{2}>: i \in J_{0}\right\}$ is a intuitionistic finite subcover of $A$.

Definition III.2. An intuitionistic subset $A$ in a intuitionistic topological space $(\tilde{X}, \tilde{\tau})$ is a intuitionistic $N$ closed in $(\tilde{X}, \tilde{\tau})$ iff for each intuitionistic open cover $\left\{G_{i}=\left\langle G_{i}^{1}, G_{i}^{2}>: i \in J\right\}\right.$ of $A$, there exists a finite set $J_{0}$ of $J$ such that $A \subseteq \bigcup_{i \in J_{0}} \operatorname{int}\left(\operatorname{el}\left(G_{i}\right)\right)$.

Example III.3. Let $X=\mathrm{P}$ and $\tilde{\tau}$ is as follows.(i) $\tilde{\varnothing}$, (ii) $\tilde{\mathrm{P}}$, (iii) $\left\langle\cup\left(a_{i}, b_{i}\right),(-\infty, c]>\right.$ where $a_{i}, b_{i} \in \mathrm{P}$ and $\left\{a_{i}: i \in J\right\}$ is bounded below and $c \in \inf \left\{a_{i}: i \in J\right\}$.

(iv) $\left\langle\bigcup\left(a_{i}, b_{i}\right), \Phi\right\rangle$ where $a_{i}, b_{i} \in \mathrm{P}$ and $\left\{a_{i}: i \in J\right\}$ is not bounded below.

$A=\langle[o, 1],(-\infty, 0)>$ is an intuitionistic $N$-closed set in $(\tilde{X}, \tilde{\tau})$.

Definition III.4. An intuitionistic topological space $(\tilde{X}, \tilde{\tau})$ is said to be intuitionistic almost regular if for each 
intuitionistic regular closed set $A$ and any intuitionistic point $\tilde{x}$ not in $A$, there exists disjoint intuitionistic open sets $U$ and $V$ such that $A \subseteq U$ and $\tilde{x} \in V$.

The following theorem characterizes intuitionistic $\mathrm{N}$-Closed spaces.

Theorem III.5. An intuitionistic subset $A$ of a intuitionistic topological space $(\tilde{X}, \tilde{\tau})$ is intuitionistic $N$ closed if and only if for each intuitionistic regular open cover of $A$ has a intuitionistic finite sub cover.

Proof. Necessity- Let $A$ be a intuitionistic $N$-closed set in a intuitionistic topological space $(\tilde{X}, \tilde{\tau})$ and $\left\{G_{i}: i \in J\right\}$ be any intuitionistic regular open cover of $A$. Therefore, $A \subseteq \bigcup_{i \in J} G_{i}=\bigcup_{i \in J} \operatorname{int}\left(\operatorname{cl}\left(G_{i}\right)\right)$ and hence $\left\{\operatorname{int}\left(e l\left(G_{i}\right)\right): i \in J\right\}$ is an intuitionistic open cover of $A$. By hypothesis, there exists a finite set $J_{0}$ of $\quad J$ such that $A \subseteq \bigcup_{i \in J_{0}} \operatorname{int}\left(\operatorname{cl}\left(\operatorname{int}\left(\operatorname{cl}\left(G_{i}\right)\right)\right)\right) \subseteq \bigcup_{i \in J_{0}} \operatorname{int}\left(\operatorname{cl}\left(G_{i}\right)\right)=\bigcup_{i \in J_{0}} G_{i}$. Thus, $A \subseteq \bigcup_{i \in J_{0}} \operatorname{int}\left(\operatorname{el}\left(G_{i}\right)\right)$

Sufficiency- Suppose that $\left\{G_{i}: i \in J\right\}$ be an open cover of A.By theorem 3.5 [3], $\operatorname{int}\left(\operatorname{el}\left(G_{i}\right)\right)$

is intuitionistic regular open set for each i.Also, $\left\{\operatorname{int}\left(\operatorname{ct}\left(G_{i}\right)\right): i \in J\right\}$ is an intuitionistic regular open cover of $A$.By hypothesis,there existys a finite set $\mathrm{J}_{0}$ of $\mathrm{J}$ such that $A \subseteq \bigcup_{i \in J_{0}} \operatorname{int}\left(c t\left(G_{i}\right)\right)$. Therefore. A is intuitionistic $N$-closed set.

Theorem III.6. An intuitionistic space $(\tilde{X}, \tilde{\tau})$ is nearly compact if and only if it is intuitionistic $N$-closed. Proof.It follows from the definitions.

Theorem III.7. In an intuitionistic topological space $(\tilde{X}, \tilde{\tau})$, the intersection of a intuitionistic $N$-closed set and a intuitionistic regular closed set is always a intuitionistic $N$-closed set.

Proof. Let A be any intuitionistic $N$-closed and B be intuitionistic regular closed subset of a intuitionistic topological space $(\tilde{X}, \tilde{\tau})$.Suppose that $\left\{G_{i}: i \in J\right\}$ is any intuitonistic regular open cover of $A \cap B$. Since $A=(A \backslash B) \cup(A \cap B)$, we have $A \subseteq(X \backslash B) \bigcup_{i \in J} G_{i}$. Therefore,

$\left\{(X \backslash B), \bigcup\left\{G_{i}: i \in J\right\}\right\}$ is a intuitionistic regular open cover of a intuitionistic $N$-closed set A in $(\tilde{X}, \tilde{\tau})$. By hypothesis,there exists a finite subset $J_{0}$ of $J$ such that $A \subseteq\left(\bigcup_{i \in J_{0}} G_{i}\right) \bigcup(X \backslash B)$. Therefore ,there exists a finite subset $J_{0}$ of $J$ such that $A \cap B \subseteq \bigcup_{i \in J_{0}} G_{i}$. Thus, $A \cap B$ is a intuitionistic $N$-closed set $\operatorname{in}(\tilde{X}, \tilde{\tau})$.

Theorem III.8. Let $A$ and $B$ be intuitionistic subsets of a intuitionistic topological space $(\tilde{X}, \tilde{\tau})$ such that $B \subseteq A$. Then the following statements hold.

(i).If $A$ is intuitionistic $N$-closed and $B$ is intuitionistic regular closed set in $(\tilde{X}, \tilde{\tau})$, then $B$ is intuitionistic $N$-closed set in $(\tilde{X}, \tilde{\tau})$.

(ii).If $A$ is intuitionistic $N$-closed and $B$ is intuitionistic regular open set in $(\tilde{X}, \tilde{\tau})$, then $A \backslash B$ is intuitionistic $N$-closed set in $(\tilde{X}, \tilde{\tau})$.

(iii).If $A$ is intuitionistic regular closed and $\$ is intuitionistic nearly compact,then $A$ is intuitionistic $N$ closed set in $\tilde{X}$.

proof-(i).Since $B \subseteq A$, we have $A \cap B=B$. Thus, by theorem III.7, $B$ is intuitionistic $N$-closed set in $(\tilde{X}, \tilde{\tau})$.

(ii).Let $A$ be a intuitionistic N-closed and $B$ be intuitionistic regular open subsets of a intuitionistic 
topological space $(\tilde{X}, \tilde{\tau})$. Suppose that $\left\{G_{i}: i \in J\right\}$ is any intuitionistic regular open cover of $A \backslash B$. Since $A=(A \backslash B) \bigcup(A \cap B)=(A \backslash B) \bigcup B$, we have $A \subseteq\left(\bigcup_{i \in J} G_{i}\right) \bigcup B$. Therefore, $\left\{\mathrm{G}_{\mathrm{i}}: \mathrm{i} \in \mathrm{J}\right\} \cup \mathrm{B}$ is a intuitionistic regular open cover of intuitionistic $N$-closed set $\mathrm{A}$ in $(\tilde{X}, \tilde{\tau})$. By hypothesis, there exists a finite subset $J_{0}$ of $J$ such that $A \backslash B \subseteq \bigcup_{i \in J_{0}} G_{i}$. Thus, $A \backslash B$ is a intuitionistic $N$-closed set.

(iii).By theorem III.6, $(\tilde{X}, \tilde{\tau})$ is intuitionistic N-closed. Since $A \subseteq X$, we have $A \cap X=A$. By (i), $A$ is intuitionistic $N$-closed set in $(\tilde{X}, \tilde{\tau})$.

Definition III.9.A function $\mathrm{f}:(\tilde{X}, \tilde{\tau}) \rightarrow(\tilde{Y}, \tilde{\sigma})$ is said to be intuitionistic almost continuous iff $f^{-1}(V)$ is intuitionistic open in $(\tilde{X}, \tilde{\tau})$ for every intuitionistic regular open set $V$ of $(\tilde{Y}, \tilde{\sigma})$.

Theorem III.10. Let $\mathrm{f}:(\tilde{X}, \tilde{\tau}) \rightarrow(\tilde{Y}, \tilde{\sigma})$ be a intuitionistic almost continuous function.Then any intuitionistic almost continuous image of a intuitionistic compact set in $(\tilde{X}, \tilde{\tau})$ is intuitionistic $N$-closed set in a $(\tilde{Y}, \tilde{\sigma})$.

Proof. Given that $\mathrm{f}:(\tilde{X}, \tilde{\tau}) \rightarrow(\tilde{Y}, \tilde{\sigma})$ is intuitionistic almost continuous.Let $A$ be intuitionistic compact set of $(\tilde{X}, \tilde{\tau})$. Let $\left\{G_{i}: i \in J\right\}$ be any intuitionistic regular open cover of $f(A)$. Since $f$ is intuitionistic almost continuous, for each $i \in J, f^{-1}\left(G_{i}\right)$ is intuitionistic open in $(\tilde{X}, \tilde{\tau})$.Also, $A \subseteq f^{-1}(f(A)) \subseteq f^{-1}\left(\bigcup_{i \in J} G_{i}\right)=\bigcup_{i \in J} f^{-1}\left(G_{i}\right)$. Therefore $\left\{f^{-1}\left(G_{i}\right): i \in J\right\}$ is a intuitionistic open cover of a intuitionistic compact set $A$.Therefore, there exists a finite number of intuitionistic regular open sets $G_{1}, G_{2}, \ldots G_{n}$ in $(\tilde{X}, \tilde{\tau})$ such that $A \subseteq \bigcup_{i=1}^{i=n} f^{-1}\left(G_{i}\right)$.

Thus, $f(A) \subseteq f\left(\bigcup_{i=1}^{n} f^{-1}\left(G_{i}\right)\right)=\left(\bigcup_{i=1}^{n} f\left(f^{-1}\left(G_{i}\right)\right)\right) \subseteq \bigcup_{i=1}^{n} G_{i}$.Thus, $f(A)$ is intuitionistic $N$-closed set in $(\tilde{Y}, \tilde{\sigma})$.

\section{Between intuitionistic $N$-Closed Sets and T_2(i).}

Definition IV.1.[6] A intuitionistic topological space is said to be $T_{2}(i)$ iff for all distinct pair of points $x, y \in X$, there exists a pair of disjoint intuitionistic open sets $U$ and $V$ such that $\tilde{x} \in U$ and $\tilde{y} \in V$.

Proposition IV.2. In a intuitionistic topological space $(\tilde{X}, \tilde{\tau})$, the intuitionistic sets $U$ and $A$ are such that $U \cap A=\Phi$ if and only if $A \subseteq U^{c}$.

Proof. Obvious.

Proposition IV.3.Let $A$ be any intuitionistic subset of a intuitionistic topological space $(\tilde{X}, \tilde{\tau})$ and $\tilde{x}$ be any intuitionistic point of $X$. Then $\tilde{x} \in \operatorname{el}(A)$ iff every intuitionistic open set containing $\tilde{x}$, intersect $A$.

Proof. Necessity- Let $\tilde{x} \in \operatorname{el}(A)$ and let $U$ be any intuitionistic open set containing $\tilde{x}$ such that $U \cap A=\Phi$ Then by theorem $4.2, A \subseteq U^{c}$ and hence $U^{c}$ is intuitionistic closed set containing $A$. But $\overline{c l}(A)$ is the smallest intuitionistic closed set containing $A$. Therefore $\operatorname{cl}(A) \subseteq U^{c}$ Now $\tilde{x} \notin U^{c}$ implies that $\tilde{x} \notin e l(A)$, a contradiction.

Sufficiency- Suppose that $\tilde{x} \notin \operatorname{ct}(A)$. By definition,there exists an intuitionistic closed set $F$ containing $A$ does not containing $\tilde{x}$. Thus $\tilde{x} \in F^{c}$ such that $F^{c} \subseteq A^{c}$ and hence $F^{c} \cap A=\Phi$. Now there exists a 
intuitionistic open set $F^{c}$ containing $\tilde{x}$, does not intersect $A$, a contradiction.Therefore $\tilde{x} \in \overline{e l}(A)$.

Lemma IV.4. Let $A$ and $B$ be any two intuitionistic sets in intuitionistic topological space $(\tilde{X}, \tilde{\tau})$. Then $\operatorname{ct}(A \cap B) \subseteq \operatorname{el}(A) \cap \operatorname{et}(B)$.

Proof. [2] By proposition 3.16, $A \subseteq \operatorname{cl}(A)$ and $B \subseteq \operatorname{cl}(B)$. Therefore, $A \cap B \subseteq \operatorname{cl}(A) \cap \operatorname{cl}(B)$ and . $\overline{c l}(A) \cap \overline{c l}(B)$ is a intuitionistic closed set containing $A \cap B$. But $\overline{c l}(A \cap B)$ is the smallest intuitionistic closed set containing $A \cap B$. Thus, $\operatorname{el}(A \cap B) \subseteq \operatorname{el}(A) \cap \operatorname{cl}(B)$.

Remark IV.5.The reversible inclusion is not true in general from the following example.

ExampleIV.6.Let $X=\{a, b\}$ and $\tilde{\tau}=\{\tilde{\Phi}, \tilde{X},\langle\{a, \varnothing\}\rangle,\langle\{b, \varnothing\}\rangle,\langle\{a, b\}\rangle,\langle\varnothing, \varnothing\rangle,\langle\varnothing, b\rangle\}$. If $A=\langle\varnothing, b\rangle$ and $B=\langle\{b, a\}\rangle$, then $A \cap B=\tilde{\Phi}=c \tilde{l}(A \cap B)$ whereas $\operatorname{ct}(A) \cap \operatorname{cl}(B)=B$.

Theorem IV.7. Let $(\tilde{X}, \tilde{\tau})$ be a $T_{2}(i)$ space.Then for every intuitionistic N-closed set $A$ in $(\tilde{X}, \tilde{\tau})$ and every point $\tilde{y} \in \tilde{X} \backslash A$, there exists a pair of disjoint intuitionistic regularly open sets $U$ and $V$ such that $\tilde{y} \in U$ and $A \subseteq V$.

Proof. Given that is a $T_{2}(i)$ space and $A$ is any intuitionistic $N$-closed set and $\tilde{y} \in \tilde{X} \backslash A$, is an arbitrary intuitionistic point in $(\tilde{X}, \tilde{\tau})$.Let $\tilde{x} \in A$ be arbitrary.Then $\tilde{x} \neq \tilde{y}$. By hypothesis, there exists disjoint intuitionistic open sets $U_{\tilde{x}}$ and $V_{\tilde{x}}$ such that $\tilde{x} \in V_{\tilde{x}}$ and $\tilde{y} \in U_{\tilde{x}}$. By theorem 3.5 [3], $\operatorname{int}\left(\operatorname{cl}\left(V_{\tilde{x}}\right)\right)$ is an intuitionistic regular open set in $(\tilde{X}, \tilde{\tau})$.Hence $\left\{\operatorname{int}\left(\mathcal{c l}_{(}\left(V_{\tilde{x}}\right)\right): \tilde{x} \in A\right\}$ is an intuitionistic regular open cover of a intuitionistic $N$-closed set $A$. Therefore, there exists a finite number of intuitionistic points $\left\{x_{1}, x_{2}, \ldots x_{n}\right\}$ in $A$ such that $A \subseteq \bigcup_{i=1}^{n} \operatorname{int}\left(e l\left(V_{x_{i}}\right)\right)$.Let $U_{0}=\bigcap_{i=1}^{n} U_{\tilde{x}_{i}}$ and $V_{0}=\bigcup_{i=1}^{n} \operatorname{int}\left(e l\left(V_{\tilde{x}_{i}}\right)\right)$ then $U_{0}$ and $V_{0}$ are intuitionistic open sets such that $\tilde{y} \in U_{0}$ and $A \subseteq V_{0}$. To prove $U_{0} \cap V_{0}=\varnothing$, we take a point $\tilde{z} \in U_{0} \cap V_{0}$. Then $\tilde{z} \in U_{\tilde{x}_{i}}$ and $\tilde{z} \in \operatorname{int}(c t)\left(V_{\tilde{x}_{i}}\right) \subseteq \operatorname{cl}\left(V_{\tilde{x}_{i}}\right)$ for some $i$. By Proposition IV.3, every intuitionistic open subset containing $\tilde{z}$ intersects $V_{\tilde{x}_{i}}$. Therefore $U_{\tilde{x}_{i}} \cap V_{\tilde{x}_{i}} \neq \tilde{\varnothing}$ for some $i$, a contradiction. Therefore $U_{0} \cap V_{0}=\tilde{\varnothing}$. Let $U=\operatorname{int}(e l)\left(U_{0}\right)$ and $V=\operatorname{int}(e l)\left(V_{0}\right)$ Then $U$ and $V$ are a pair of disjoint intuitionistic regularly open sets such that $\tilde{y} \in U$ and $A \subseteq V$.

Corollary IV.8.Every intuitionistic nearly compact, $T_{2}(i)$ space is almost regular.

Proof. By (iii) of theorem III.8, every intuitionistic regular closed set of a intuitionistic nearly compact space is intuitionistic $N$-closed and hence by theorem $4.7,(\tilde{X}, \tilde{\tau})$ is intuitionistic almost regular space.

Lemma IV.9. In a intuitionistic topological space $(\tilde{X}, \tilde{\tau})$, intersection of any two intuitionistic regular open sets is a intuitionistic regular open set in $(\tilde{X}, \tilde{\tau})$.

Proof. Let $A$ and $B$ be any two intuitionistic regular open sets in a intuitionistic space $(\tilde{X}, \tilde{\tau})$.

Therefore,they are intuitionistic open sets in $(\tilde{X}, \tilde{\tau})$ and so their intersections.

Thus, $A \cap B=\operatorname{int}(A \cap B) \subseteq \operatorname{int}(\operatorname{ct}(A \cap B))$.On the other hand, by lemma IV.4, $\operatorname{ct}(A \cap B) \subseteq \operatorname{el}(A) \cap \operatorname{et}(B)$.

Therefore, int $(\operatorname{cl}(A \cap B)) \subseteq \operatorname{int}(\operatorname{cl}(A) \cap \operatorname{ct}(B))=\operatorname{int}(\operatorname{ct}(A)) \bigcap \operatorname{int}(\operatorname{ct}(B))=A \cap B$.

Hence $\operatorname{int}(\operatorname{cl}(A \cap B))=A \cap B$. Thus, intersection of any two intuitionistic regular open sets is a intuitionistic regular open set in $(\tilde{X}, \tilde{\tau})$. 
Theorem IV.10. Let $(\tilde{X}, \tilde{\tau})$ be a $T_{2}(i)$ space.Then for any two disjoint intuitionistic N-closed sets $A$ and $B$, there exists a pair of disjoint intuitionistic regular open sets $U$ and $V$ such that $A \subseteq U$ and $B \subseteq V$.

Proof. Given that $A$ and $B$ are any two disjoint intuitionistic $N$-closed sets in $(\tilde{X}, \tilde{\tau})$.Let $\tilde{y}$ be any point of $B$.Then $\tilde{y} \in X \backslash A$. By theorem IV.7,there exists disjoint intuitionistic regularly open sets $U_{\tilde{y}}$ and $V_{\tilde{y}}$ such that $\tilde{y} \in V_{\tilde{y}}$ and $A \subseteq U_{\tilde{y}}$. Then the family $\left\{V_{\tilde{y}}: \tilde{y} \in B\right\}$ is an intuitionistic regular open cover for a intuitionistic $N$-closed set $B$ in $(\tilde{X}, \tilde{\tau})$. Therefore,there exists a finite number of intuitionistic points $\tilde{y}_{1}, \tilde{y}_{2}, \ldots \tilde{y}_{n}$ in $B$ such that $B \subseteq \bigcup_{i=1}^{n} V_{\tilde{y}_{i}}$. Let $U=\bigcap_{i=1}^{n} U_{\tilde{y}_{i}}$ and $V_{0}=\bigcup_{i=}^{n} V_{\tilde{y}_{i}}$. Then $A \subseteq U$ and $B \subseteq V_{0}$ such that $U \cap V_{0}=\tilde{\varnothing}$. If we define $V=\operatorname{int}(c l)\left(V_{0}\right)$, then $U$ and $V$ are disjoint regular open sets such that $A \subseteq U$ and $B \subseteq V$.

Theorem IV.11. Every intuitionistic singleton set $\{\tilde{x}\}$ of $(\tilde{X}, \tilde{\tau})$ is intuitionistic $\mathrm{N}$-closed subset in $(\tilde{X}, \tilde{\tau})$.

Proof. Let $\{\tilde{x}\}$ be any intuitionistic point of $(\tilde{X}, \tilde{\tau})$ and suppose that $\left\{G_{i} \in \tilde{\tau}: i \in J\right\}$ is any intuitionistic open cover of $\{\tilde{x}\}$. Therefore $\quad\{\tilde{x}\} \subseteq \bigcup_{i \in J} G_{i}$ and hence $\{\tilde{x}\} \in G_{i}$ for some $i$. Thus, $\{\tilde{x}\} \in G_{i}=\operatorname{int}\left(G_{i}\right) \subseteq \operatorname{int}\left(\operatorname{el}\left(G_{i}\right)\right)$ for some $i$ and hence $\{\tilde{x}\}$ is intuitionistic N -closed subset of $(\tilde{X}, \tilde{\tau})$.

Corollary IV.12.A intuitionistic topological space $(\tilde{X}, \tilde{\tau})$ is said to be $T_{2}(i)$ iff for any two disjoint intuitionistic $N$-closed sets $A$ and $B$, there exists a pair of disjoint intuitionistic regular open sets $U$ and $V$ such that $A \subseteq U$ and $B \subseteq V$.

Proof. Necessity-It follows from theorem 4.10.

Sufficiency- Let $\{\tilde{x}\}$ and $\{\tilde{y}\}$ be any two intuitionistic points of $(\tilde{X}, \tilde{\tau})$ such that $\{\tilde{x}\} \neq\{\tilde{y}\}$. By theorem $4.11,\{\tilde{x}\}$ and $\{\tilde{y}\}$ are two disjoint intuitionistic $N$-closed subsets of $(\tilde{X}, \tilde{\tau})$.By hypothesis,there exists disjoint intuitionistic regular open sets $U$ and $V$ in $(\tilde{X}, \tilde{\tau})$ such that $\{\tilde{x}\} \subseteq U$ and $\{\tilde{y}\} \subseteq V$. Thus $(\tilde{X}, \tilde{\tau})$ is $T_{2}(i)$.

Theorem IV.13. In a intuitionistic topological space the following statements are equivalent.

(i). $(\tilde{X}, \tilde{\tau})$ is intuitionistic almost regular space.

(ii). For each intuitionistic point $\tilde{x} \in \tilde{X}$, and each intuitionistic regular-open set $V$ in $(\tilde{X}, \tilde{\tau})$ containing $\tilde{x}$, there exists a intuitionistic regular open set $U$ in such that $\tilde{x} \in U \subseteq \overline{l l}(U) \subseteq V$.

(iii).For each intuitionistic point $\tilde{x} \in \tilde{X}$, and each intuitionistic open set $U$ in $(\tilde{X}, \tilde{\tau})$ containing $\tilde{x}$, there exists a intuitionistic regular open set $V$ in $(\tilde{X}, \tilde{\tau})$ such that $\tilde{x} \in V \subseteq \operatorname{ct}(V) \subseteq \operatorname{int}(\operatorname{ct}(U))$.

(iv).For each intuitionistic point $\tilde{x} \in \tilde{X}$, and each intuitionistic open set $U$ in $(\tilde{X}, \tilde{\tau})$ containing $\tilde{x}$, there exists a intuitionistic open set $V$ in such that $\tilde{x} \in V \subseteq \operatorname{cl}(V) \subseteq \operatorname{int}(\operatorname{cl}(U))$.

(v). For every intuitionistic regular-closed set $A$ in $(\tilde{X}, \tilde{\tau})$ and each intuitionistic point $\tilde{x} \notin A$, there exists intuitionistic open sets $U$ and $V$ such that $\tilde{x} \in U, A \subseteq V$ and $\operatorname{cl}(U) \bigcap \operatorname{cl}(V)=\tilde{\Phi}$.

proof.(i) $\Rightarrow$ (ii).Let $\tilde{x} \in \tilde{X}$, be any intuitionistic point and $V$ be any intuitionistic regular-open set in containing $\tilde{x}$. Then $V^{c}$ is an intuitionistic regular-closed set in $(\tilde{X}, \tilde{\tau})$ does not containing $\tilde{x}$. By hypothesis,there exists disjoint intuitionistic open sets $U_{1}$ and $U_{2}$ in $(\tilde{X}, \tilde{\tau})$ such that $\{\tilde{x}\} \subseteq U_{1}$ and $V^{c} \subseteq U_{2} . \quad$ Therefore, $\operatorname{cl}\left(U_{1}\right) \cap U_{2}=\tilde{\Phi} \quad$ implies that $\quad c \tilde{l}\left(U_{1}\right) \subseteq U_{2}^{c} \subseteq V$. 
Moreover, $U_{1}=\operatorname{int}\left(U_{1}\right) \subseteq \operatorname{int}\left(\operatorname{ct}\left(U_{1}\right)\right) \subseteq \operatorname{et}\left(U_{1}\right) \subseteq V$. If we define $U=\operatorname{int}\left(\operatorname{ct}\left(U_{1}\right)\right)$, then $U$ is a intuitionistic regular-open set such that $\tilde{x} \in U \subseteq \overline{e l}(U) \subseteq V$.

(ii) $\Rightarrow$ (iii).Let $\tilde{x}$ be any intuitionistic point in $(\tilde{X}, \tilde{\tau})$ and $U$ be any intuitionistic open set in $(\tilde{X}, \tilde{\tau})$ containing $\tilde{x}$. By theorem $3.5[3]$, $\operatorname{int}(\operatorname{ct}(U))$ is intuitionistic regular open set in $(\tilde{X}, \tilde{\tau})$ containing $\tilde{x}$. By hypothesis,there exists a intuitionistic regular open set $\mathrm{V}$ in such that $\tilde{x} \in V \subseteq \overline{e l}(V) \subseteq \operatorname{int}(\operatorname{cl}(U))$.

(iii) $\Rightarrow$ (iv). Obvious.

(iv) $\Rightarrow(\mathbf{v})$. Let $\tilde{x}$ be any intuitionistic point in $(\tilde{X}, \tilde{\tau})$ and $A$ be any intuitionistic regular closed set in $(\tilde{X}, \tilde{\tau})$ does not containing $\tilde{x}$. Then $A^{c}$ is a intuitionistic regular open set in $(\tilde{X}, \tilde{\tau})$ and hence intuitionistic open set in $(\tilde{X}, \tilde{\tau})$ containing $\tilde{x}$. By hypothesis, there exists an intuitionistic open set $V_{1}$ containing $\tilde{x}$ such that $V_{1} \subseteq c l\left(V_{1}\right) \subseteq \operatorname{int}\left(c l\left(A^{c}\right)\right)=A^{c}$. Therefore, $A \subseteq\left[\overline{c l}\left(V_{1}\right)\right]^{c}$. In a similar way,if we apply hypothesis to the intuitionistic open set $V_{1}$, then there exists an intuitionistic open set $V_{2}$ containing $\tilde{x}$ such that $\overline{c l}\left(V_{2}\right) \subseteq \operatorname{int}\left(c l\left(V_{1}\right)\right)$. If we define $U=V_{2}$ and $\mathrm{V}=\left[\overline{c l}\left(V_{1}\right)\right]^{c}$, then $U$ and $V$ are intuitionistic open sets such that $\tilde{x} \in U, A \subseteq V$ and $\operatorname{cl}(U) \cap \operatorname{cl}(V)=\tilde{\Phi}$.

(v) $\Rightarrow$ (i). Let $\tilde{x}$ be any intuitionistic point in $(\tilde{X}, \tilde{\tau})$ and $A$ be any intuitionistic regular closed set does not containing $\tilde{x}$. By hypothesis, there exists intuitionistic open sets $U$ and $V$ in $(\tilde{X}, \tilde{\tau})$ such that $\tilde{x} \in U$ and $A \subseteq V, \operatorname{cl}(U) \bigcap \operatorname{cl}(V)=\tilde{\Phi}$. Hence $U \cap V=\Phi$ Thus, $(\tilde{X}, \tilde{\tau})$ is a intuitionistic almost regular space.

Theorem IV.14. An intuitionistic topological space $(\tilde{X}, \tilde{\tau})$ is almost regular if and only if for any intuitionistic $N$-closed set $A$ and intuitionistic regular closed set $B$ in $(\tilde{X}, \tilde{\tau})$ such that $A \cap B=\tilde{\Phi}$, there exists intuitionistic open sets $U$ and $V$ in $(\tilde{X}, \tilde{\tau})$ such that $A \subseteq U, B \subseteq V$ and $\operatorname{ct}(U) \cap \operatorname{ct}(V)=\tilde{\Phi}$.

Proof. Necessity- Let $A$ be any intuitionistic $N$-closed set and $B$ be any intuitionistic regular closed set in $(\tilde{X}, \tilde{\tau})$.By hypothesis and by theorem IV.13(v), for each intuitionistic point $\tilde{x} \in A$, (and hence $\tilde{x} \notin B$,) there exists intuitionistic open sets $U_{\tilde{x}}$ and $V_{\tilde{x}}$ such that $\tilde{x} \in U_{\tilde{x}}$ and $B \subseteq V_{\tilde{x}}$ and $\operatorname{ct}\left(U_{\tilde{x}}\right) \cap \operatorname{ct}\left(V_{\tilde{x}}\right)=\tilde{\Phi}$ .Then the family $\left\{U_{\tilde{x}}: \tilde{x} \in A\right\}$ covers $A$. Since $A$ is intuitionistic $N$-closed set in $(\tilde{X}, \tilde{\tau})$, there exists a finite number of intuitionistic points $\tilde{x}_{1}, \tilde{x}_{2}, \ldots \tilde{x}_{n}$ in $A$ such that $A \subseteq \bigcup_{i=1}^{n} \operatorname{int}\left(\operatorname{ct}\left(U_{\bar{x}_{i}}\right)\right)$.If we define $U=\bigcup_{i=1}^{n} \operatorname{int}\left(e l\left(U_{\Psi_{i}}\right)\right)$ and $V=\bigcap_{i=1}^{n} V_{x_{i}}$. Then $U$ and $V$ are intuitionistic open sets such that $A \subseteq U, B \subseteq V$ and $\overline{c l}(U) \bigcap \overline{c t}(V)=\tilde{\Phi}$.

Sufficiency-It follows from theorem 4.13 ( v) $\Rightarrow$ (i).

\section{References .}

[1] Dogan Coker; "A Note On Intutionistic Sets And Intutionistic Points." Tr. J. Of Mathematics 20 (1996), 343-351.

[2] Dogan Coker; "An Introduction to Intutionistic Topological Spaces." Busefal 81, 51-56 (2000).

[3] M.Lellis Thivagar,M.Anbuchelvi,Saeid Jafari; "Note on Intuitionistic Compactness." (Communicated).

[4] T.Noiri; " $N$-closed sets and some separation axioms." Annales de la Societe Scientifique de Bruexelles, T. 88,II,pp.195-199 (1974).

[5] M.K.Singal and Shashi Prabha Arya; "On Almost regular spaces." Glasnik Mat., 4 (24), 89-99, 1969.

[6] Sadik Bayhan and Dogan Coker; "On Separation Axioms in Intutionistic Topological Spaces." IJMMS (2001) 621 -630. 